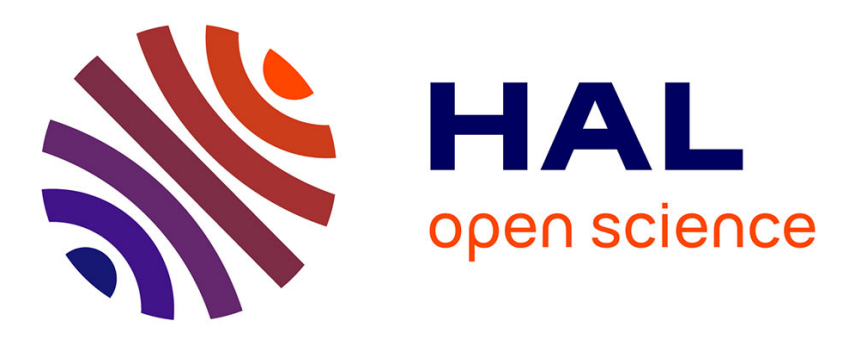

\title{
Sparse Jurdjevic-Quinn stabilization of dissipative systems
}

Marco Caponigro, Benedetto Piccoli, Francesco Rossi, Emmanuel Trélat

\section{To cite this version:}

Marco Caponigro, Benedetto Piccoli, Francesco Rossi, Emmanuel Trélat. Sparse Jurdjevic-Quinn stabilization of dissipative systems. Automatica, 2017, 86, pp.110-120. 10.1016/j.automatica.2017.08.012 . hal-01397843v3

\section{HAL Id: hal-01397843 https://hal.science/hal-01397843v3}

Submitted on 24 May 2017

HAL is a multi-disciplinary open access archive for the deposit and dissemination of scientific research documents, whether they are published or not. The documents may come from teaching and research institutions in France or abroad, or from public or private research centers.
L'archive ouverte pluridisciplinaire HAL, est destinée au dépôt et à la diffusion de documents scientifiques de niveau recherche, publiés ou non, émanant des établissements d'enseignement et de recherche français ou étrangers, des laboratoires publics ou privés. 


\title{
Sparse Jurdjevic-Quinn stabilization of dissipative systems
}

\author{
Marco Caponigro* $\quad$ Benedetto Piccoli ${ }^{\dagger} \quad$ Francesco Rossi ${ }^{\ddagger} \quad$ Emmanuel Trélat ${ }^{\S}$
}

May 24, 2017

\begin{abstract}
For control-affine systems with a proper Lyapunov function, the classical Jurdjevic-Quinn procedure (see [22]) gives a well-known and widely used method for the design of feedback controls that asymptotically stabilize the system to some invariant set. In this procedure, all controls are in general required to be activated, i.e. nonzero, at the same time.

In this paper we give sufficient conditions under which this stabilization can be achieved by means of sparse feedback controls, i.e., feedback controls having the smallest possible number of nonzero components. We thus obtain a sparse version of the classical Jurdjevic-Quinn theorem.

We propose three different explicit stabilizing control strategies, depending on the method used to handle possible discontinuities arising from the definition of the feedback: a time-varying periodic feedback, a sampled feedback, and a hybrid hysteresis.

We illustrate our results by applying them to opinion formation models, thus recovering and generalizing former results for such models.
\end{abstract}

\section{Introduction and main result}

\subsection{The context}

Let $n$ and $m$ be positive integers, let $f$ and $g_{i}, i=1, \ldots, m$ be smooth vector fields defined on $\mathbb{R}^{n}$, and let $\mathbb{U}$ be a convex subset of $\mathbb{R}^{m}$ containing a neighborhood of the origin. We consider the control-affine system in $\mathbb{R}^{n}$

$$
\dot{x}(t)=f(x(t))+\sum_{i=1}^{m} u_{i}(t) g_{i}(x(t)),
$$

where the control $u=\left(u_{1}, \ldots, u_{m}\right)$ takes its values in $\mathbb{U}$. We assume the uncontrolled system (i.e., with $u \equiv 0)$ to be dissipative, meaning that there exists a smooth function $V: \mathbb{R}^{n} \rightarrow \mathbb{R}$ such that:

- $V$ is radially unbounded (or proper), i.e., $V^{-1}((-\infty, \ell])$ is compact for every $\ell \in \mathbb{R}$;

- $L_{f} V(x) \leq 0$ for every $x \in \mathbb{R}^{n}$.

\footnotetext{
${ }^{*}$ Conservatoire National des Arts et Métiers, Équipe M2N, 292 rue Saint-Martin, 75003, Paris, France. (marco.caponigro@cnam.fr)

${ }^{\dagger}$ Department of Mathematical Sciences and Center for Computational and Integrative Biology, Rutgers University, Camden, NJ 08102, USA (piccoli@camden.rutgers.edu)

${ }^{\ddagger}$ Aix Marseille Université, CNRS, ENSAM, Université de Toulon, LSIS, Marseille, France. (francesco.rossi@lsis.org)

${ }^{\S}$ Sorbonne Universités, UPMC Univ. Paris 6, CNRS UMR 7598, Laboratoire Jacques-Louis Lions and Institut Universitaire de France, 4 place Jussieu, 75005, Paris, France (emmanuel.trelat@upmc.fr)
} 
According to the well-known Jurdjevic-Quinn theorem (see [22]), if we assume that $f(0)=0$ and that

$$
\left\{x \in \mathbb{R}^{n} \mid L_{f} V(x)=0 \text { and } L_{f}^{k} L_{g_{i}} V(x)=0, \text { for } i=1, \ldots, m, k \in \mathbb{N}\right\}=\{0\},
$$

then the smooth feedback defined by

$$
u(x)=-\left(L_{g_{1}} V(x), L_{g_{2}} V(x), \ldots, L_{g_{m}} V(x)\right)
$$

globally asymptotically stabilizes the system (1) to 0. A more general version gives the convergence to some invariant set. The convergence is established by the LaSalle invariance principle. This famous result has been widely used, in various contexts, ranging from the control of mechanical systems (see for instance $[18,19,27])$ to mathematical biology (see, e.g., [3]).

In the above strategy, all components of the control are in general active i.e., they take non-zero values. We address here the following question: is it possible to design a similar Jurdjevic-Quinn stabilizing feedback strategy in which only a minimal number of controls are active at each instant of time?

This question is inspired by the works $[9,10]$ introducing the notion of sparse control. The term "sparse" may refer to components or to time.

A componentwise-sparse control has only at most one active component at each instant of time. Componentwise sparsity is motivated by many applications: when dealing with high-dimensional problems, that is when both $n \gg 1$ and $m \gg 1$, it may be inadequate to implement a control having $m$ active components. It is therefore natural to seek controls achieving the same goal with less active components. This is the case for instance when we want only one leader to act on a whole crowd (such as a dog with a flock of sheep), or more generally when feasible control strategies are required to focus on a small number of agents at each time (see [1, 2, 6, 7, 20, 33]).

A problem for such a componentwise-sparse control is that it may chatter, i.e., it may change active component infinitely many times over a compact time-interval; such a chattering phenomenon may cause some theoretical, numerical and practical difficulties. In particular, chattering is an obstacle to well-posedness and convergence of numerical schemes (see [34]). Time-sparsity was then introduced in $[9,10]$ to avoid these unwanted phenomena. A time-sparse control, indeed, has a minimal gap between two switchings. In this paper, we will enforce time-sparsity by using either time-sampling or hysteresis.

The motivation that we have in mind is to address the control of large groups of interacting agents, by means of control strategies that are both as simple and sparse as possible. In Section 3.2, we will then test the sparse control strategies that we develop throughout on classical examples of opinion dynamics.

\subsection{Sparse feedback stabilization strategies}

We provide hereafter three different control strategies to achieve stabilization by using a sparse Jurdjevic-Quinn controller, mimicking the form (2). Starting from this idea, our aim is to achieve sparse stabilization, by choosing sparse controls of the form $u_{i}(x)=-L_{g_{i}} V(x)$ for some $i \in\{1, \ldots, m\}$, while $u_{j}(x)=0$ for $j \neq i$. The key aspect for achieving sparse stabilization is to determine the strategy to switch from one active component of the control to another one. Indeed, discontinuity issues in the definition of sparse stabilizers arise naturally, as shown for instance in [5, 9, 10, 11], see also Section 3.1 of this paper. Here we develop three different approaches to deal with discontinuous feedbacks, each of them leading to a different kind of sparse stabilizer: a time-varying periodic feedback, a sampled feedback, and a hybrid feedback. 
Let us define the three strategies that we will consider.

The absence of continuous feedback stabilizers is a classical matter in control (as in [8]) and a classical approach [31, 29] is the introduction of time-varying periodic with respect to time feedback controls (see also [14, 28] or [15, section 11.2]). In this spirit, we consider a first strategy, as follows. Throughout the article, we denote by $e_{i}$ the unitary vector in the $i$-th variable.

\section{Strategy 1: Sparse time-varying periodic feedback.}

Fix the sampling time $\tau>0$ and the final control time $T>0$. For the initial state $x_{0} \in \mathbb{R}^{n}$, consider the unique trajectory $x(t)$ of $(1)$ with the time-varying feedback control $u(t, x)$ defined as follows:

- for each time interval $[(k m+i-1) \tau,(k m+i) \tau) \cap[0, T]$ for some $k \in \mathbb{N}$ and $i=1, \ldots, m$, apply the feedback control

$$
u(t, x)=-L_{g_{i}} V(x) e_{i}
$$

- for $t \geq T$, apply the zero control $u(t, x)=0$.

In our second sampling approach, we discretize the time horizon and we apply a fixed control $u_{i}$ on each interval. Such a control is chosen with a steepest descent approach, by maximizing the instantaneous decrease of $V$ at the beginning of the sampling interval.

\section{Strategy 2: Sampled sparse feedback.}

Consider the component-wise sparse feedback defined at any $x \in \mathbb{R}^{n}$ by

$$
u(x)=-L_{g_{i}} V(x) e_{i}
$$

where $i \in\{1, \ldots, m\}$ is the smallest integer such that

$$
\left|L_{g_{i}} V(x)\right| \geq\left|L_{g_{j}} V(x)\right|, \quad \forall j \neq i .
$$

Fix a sampling time $\tau>0$. Then consider the sampling solution associated with $u$ and the sampling time $\tau$, namely the solution of

$$
\dot{x}(t)=f(x(t))+\sum_{i=1}^{m} u_{i}(x(k \tau)) g_{i}(x(t)), \quad t \in[k \tau,(k+1) \tau],
$$

with $k \in \mathbb{N}$.

The notion of stabilization associated with sampling solutions is the stabilization in the sampleand-hold sense (see for instance [12, Section 7]).

Definition 1.1. Let $U \subset \mathbb{R}^{m}$, let $F: \mathbb{R}^{n} \times U \rightarrow \mathbb{R}^{n}$ be continuous and locally Lipschitz in $x$, uniformly on compact subsets of $\mathbb{R}^{n} \times U$, with $F(\bar{x}, 0)=0$. We say that a feedback $u: \mathbb{R}^{n} \rightarrow U$ stabilizes the system $\dot{x}=F(x, u(x))$ to $\bar{x}$ in the sample-and-hold sense if for every $r>0$ and $R>0$ there exists $\tau>0$ and $T>0$ depending only on $r$ and $R$ and $C>0$ depending only on $R$ such that for any $x_{0} \in B_{R}(\bar{x})$ the sampled solution of $\dot{x}=F(x, u(x)), x(0)=x_{0}$, with sampling time $\tau$ satisfies $|x(t)| \leq C$ for every $t \geq 0$ and $x(t) \in B_{r}(\bar{x})$ for every $t \geq T$.

Finally, we consider an hybrid approach based on hysteresis: we choose a component of the control $u_{i}$ maximizing the instantaneous decrease of $V$. This component is the only active one while it satisfies the lower threshold condition $\left|L_{g_{i}} V\right|>(1-\varepsilon)\left|L_{g_{j}} V\right|$ for any $j \neq i$. When such lower threshold is reached, the control switches to the new control maximizing the instantaneous decrease of $V$. 


\section{Strategy 3: Sparse feedback with hysteresis.}

Fix $\varepsilon \in(0,1)$ and apply the following algorithm to define the trajectory $x(t)$ of the system:

- Initialize: $n=0$ and $t_{0}=0$.

- While $t_{n}<+\infty$ apply Step $n$ : At time $t_{n}$ choose $i=1, \ldots, m$ being the smallest integer such that

$$
\left|L_{g_{i}} V\left(x\left(t_{n}\right)\right)\right| \geq\left|L_{g_{j}} V\left(x\left(t_{n}\right)\right)\right|, \quad \text { for every } j \neq i .
$$

- If $\left|L_{g_{i}} V\left(x\left(t_{n}\right)\right)\right| \geq 2 t_{n}^{-1}$, define the switching time $t_{n+1}$ as the infimum of times $t \in\left[t_{n},+\infty\right)$ such that the unique solution $y(t)$ of $\dot{y}=f(y)-L_{g_{i}} V(y) g_{i}(y)$ with $y\left(t_{n}\right)=x\left(t_{n}\right)$ satisfies

$$
\left|L_{g_{i}} V(y(t))\right| \leq t^{-1} \quad \text { or } \quad\left|L_{g_{i}} V(y(t))\right| \leq(1-\varepsilon)\left|L_{g_{j}} V(y(t))\right|, \quad \text { for some } j \neq i,
$$

with the convention that $t_{n+1}=+\infty$ if the solution satisfies

$\left|L_{g_{i}} V(y(t))\right|>t^{-1} \quad$ and $\quad\left|L_{g_{i}} V(y(t))\right|>(1-\varepsilon)\left|L_{g_{j}} V(y(t))\right|, \quad$ for every $j \neq i, t \geq t_{n}$.

Define the control $u(t, x)=-L_{g_{i}} V(x) e_{i}$ and the corresponding trajectory $x(t)$ on the interval $\left[t_{n}, t_{n+1}\right)$.

- If $\left|L_{g_{i}} V\left(x\left(t_{n}\right)\right)\right|<2 t_{n}^{-1}$, define the switching time $t_{n+1}$ as the infimum of times $t \in\left[t_{n},+\infty\right)$ such that the unique solution $y(t)$ of $\dot{y}=f(y)$ with $y\left(t_{n}\right)=x\left(t_{n}\right)$ satisfies

$$
\left|L_{g_{j}} V(y(t))\right| \geq 4 t^{-1}, \quad \text { for some } j=1, \ldots, m,
$$

with the convention that $t_{n+1}=+\infty$ if the solution satisfies

$$
\left|L_{g_{j}} V(y(t))\right|<4 t^{-1} \quad \text { for all } j=1, \ldots, m, t \geq t_{n} .
$$

Define the control $u(t, x)=0$ and the corresponding trajectory $x(t)$ on the interval $\left[t_{n}, t_{n+1}\right)$.

- If $t_{n+1}<+\infty$, pass from Step $n$ to Step $n+1$.

\subsection{Main results}

Under suitable assumptions, the three above sparse control strategies asymptotically stabilize the control system (1).

Theorem 1.1. Assume that there exists a smooth function $V: \mathbb{R}^{n} \rightarrow \mathbb{R}$ such that

- $V$ is radially unbounded (or proper), i.e., $V^{-1}((-\infty, \ell])$ is compact for every $\ell \in \mathbb{R}$;

- $L_{f} V(x) \leq 0$ for every $x \in \mathbb{R}^{n}$.

Let

$$
\mathcal{Z}=\left\{x \in \mathbb{R}^{n} \mid L_{f} V(x)=L_{g_{i}} V(x)=0, \text { for every } i=1, \ldots, m\right\},
$$

and $\Omega$ be the largest subset of $\mathcal{Z}$ that is invariant under the flow of $\dot{x}=f(x)$. Then:

(i) If $\Omega$ is locally attractive, then, for each initial state $x_{0} \in \mathbb{R}^{n}$, there exist $\tau_{1}>0$ and $T>0$ such that, for every $\tau \in\left(0, \tau_{1}\right)$, Strategy 1 with sampling time $\tau$ and final control time $T$ asymptotically stabilizes the control system (1) to $\Omega$. 
(ii) If $\mathcal{Z}=\{\bar{x}\}$ for some $\bar{x} \in \mathbb{R}^{n}$, then, for every initial state $x_{0} \in \mathbb{R}^{n}$, there exists $\tau_{2}>0$ such that, for every $\tau \in\left(0, \tau_{2}\right)$, Strategy 2 with sampling time $\tau$ asymptotically stabilizes the control system (1) to $\bar{x}$, in the sample-and-hold sense.

(iii) For every $\varepsilon \in(0,1)$, Strategy 3 asymptotically stabilizes the control system (1) to $\Omega$.

Moreover $\tau_{1}$ and $\tau_{2}$ can be chosen uniformly with respect to all initial conditions belonging to an arbitrary compact subset of $\mathbb{R}^{n}$.

This theorem is proved in Sections 2. More precisely, the convergence results are established for Strategy 1 in Proposition 2.1 (Section 2.1), for Strategy 2 in Proposition 2.2 (Section 2.2), and for Strategy 3 in Proposition 2.3 (Section 2.3), and more details and comments are provided, as well as some examples showing sharpness of the assumptions.

Let us briefly comment on advantages and drawbacks of each strategy. Strategy 1 requires local attractivity of the set $\Omega$ and, in order to design the control, the derivatives of $V(x)$ are required to be computed at any instant of time. Strategy 2 applies if the set $\mathcal{Z}$ reduces to a single point, but the evaluation of $V(x(t))$ is only required to be performed at discrete times $n \tau$, for $n \in \mathbb{N}$. The stabilization to $\mathcal{Z}$ is realized in the sample-and-hold sense (see Definition 1.1 below). Strategy 3 stabilizes the system for any parameter $\varepsilon$, and hence it is in general more robust that Strategies 1 and 2; however it is required to evaluate $V(x(t))$ at any instant of time along the trajectories.

In the definition of $\mathcal{Z}$ in Theorem 1.1, only first-order Lie derivatives of $V$ were considered. Let us now show how to generalize to higher-order derivatives, as in the classical Jurdjevic-Quinn Theorem. We say that the control system (1) satisfies the Weak Jurdjevic-Quinn Condition if there exists $l \geq 0$ such that

$$
\left\{x \in \mathbb{R}^{n} \mid L_{f} V(x)=0 \text { and } L_{f}^{k} L_{g_{i}} V(x)=0 \text {, for } i=1, \ldots, m, k \leq l\right\}=\{0\} .
$$

Of course, in the above condition 0 could be replaced with any point $\bar{x} \in \mathbb{R}^{n}$. Such a condition is sufficient (see for instance [23, Proposition 4.1 and Theorem 4.1]) for the existence of a Control Lyapunov Function for (1), that is a smooth scalar function $\mathcal{V}$ such that, if $L_{g_{i}} \mathcal{V}=0$ for every $i=1, \ldots, m$, then $L_{f} \mathcal{V}<0$ for $x \neq 0$. Then, one can apply Theorem 1.1 to the Lyapunov function $\mathcal{V}$ with $\Omega=\mathcal{Z}=\{0\}$, yielding the following result.

Corollary 1.2. Assume that the control system (1) satisfies the Weak Jurdjevic-Quinn Condition (8). Then there exists a smooth function $\mathcal{V}: \mathbb{R}^{n} \rightarrow \mathbb{R}$ such that:

(i) If 0 is locally attractive then, for every initial state $x_{0} \in \mathbb{R}^{n}$, there exist $\tau_{1}>0$ and $T>0$ such that, for every $\tau \in\left(0, \tau_{1}\right)$, Strategy 1 applied to $\mathcal{V}$ with sampling time $\tau$ and final control time $T$ asymptotically stabilizes the control system (1) to 0 ;

(ii) For every initial state $x_{0} \in \mathbb{R}^{n}$, there exists $\tau_{2}>0$ such that, for every $\tau \in\left(0, \tau_{2}\right)$, Strategy 2 applied to $\mathcal{V}$ with sampling time $\tau$ asymptotically stabilizes the control system (1) to 0 in the sample-and-hold sense.

(iii) For every $\varepsilon \in(0,1)$, Strategy 3 applied to $\mathcal{V}$ asymptotically stabilizes the control system (1) to 0.

Moreover $\tau_{1}$ and $\tau_{2}$ can be chosen uniformly with respect to all initial conditions belonging to an arbitrary compact subset of $\mathbb{R}^{n}$. 
For further remarks on the Weak Jurdjevic-Quinn Condition, see e.g. [19, Remark 3.5] and references therein.

Remark 1.1. In the definition of our strategies, we assume that the control $u_{i}=-L_{g_{i}} V(x) e_{i}$ always belongs to the set $\mathbb{U}$ of admissible controls. If this is not the case, since $\mathbb{U}$ is a closed subset containing a neighborhood of the origin, one can always replace the definition of the control with $u_{i}=\sigma\left(-L_{g_{i}} V(x) e_{i}\right)$, where $\sigma$ is the saturation operator

$$
\sigma(u)= \begin{cases}u & \text { if } u \in \mathbb{U} \\ \sup \{\lambda \in[0,1] \mid \lambda u \in \mathbb{U}\} u & \text { otherwise. }\end{cases}
$$

Our proofs obviously withstand such a modification. Indeed, for each control $g_{i}$, define the function $k_{i}(x):=\sqrt{\frac{\sigma\left(-L_{g_{i}} V(x)\right)}{-L_{g_{i}} V(x)}}$. Observe that such function is Lipschitz, since $L_{g_{i}} V$ is Lipschitz and $\sigma(u)$ is Lipschitz as a consequence of convexity of $\mathbb{U}$; moreover, the square root is a Lipschitz function away from 0 , that is ensured in our case as a consequence of $\sigma\left(-L_{g_{i}} V(x)\right)=-L_{g_{i}} V(x)$ around $-L_{g_{i}} V(x)=0$ and of boundedness of $L_{g_{i}} V(x)$. Then, define the Lipschitz vector field $\tilde{g}_{i}(x)=k_{i}(x) g_{i}(x)$ and apply one of the strategies 1,2 , or 3 to the system

$$
\dot{x}(t)=f(x(t))+\sum_{i=1}^{m} v_{i}(t) \tilde{g}_{i}(x(t)),
$$

with the same function $V$ and no constraint on the space of controls $v \in \mathbb{R}^{m}$. Then, Theorem 1.1 applied to (9) ensures that the trajectory $x(t)$ given by the chosen Strategy converges to $\Omega$, either practically or in the sample-and-hold sense or asymptotically. Observe that $\Omega$ does not change when considering vector fields $g_{i}$ or $\tilde{g}_{i}$.

We now observe that such stabilizing trajectory $x(t)$ coincides with the trajectory given by the same Strategy applied to the original system (1), when replacing the control $-L_{g_{i}} V(x) e_{i}$ with $\sigma\left(-L_{g_{i}} V(x) e_{i}\right)$. Indeed, the trajectory $x(t)$ defined above satisfies

$$
\dot{x}=f(x)-\left(L_{\tilde{g}_{i}} V(x)\right) \tilde{g}_{i}(x)=f(x)-\left(k_{i}(x) L_{g_{i}} V(x)\right)\left(k_{i}(x) g_{i}(x)\right)=f(x)+\sigma\left(-L_{g_{i}} V(x)\right) g_{i}(x) .
$$

Since $x(t)$ converges to $\Omega$, we have proved that our Strategy provides convergence even when replacing the control $u$ with the saturation operator $\sigma(u)$.

The structure of the paper is the following: Section 2 hereafter is devoted to prove the main results, and give more details about the three strategies. We illustrate our results in Section 3: in Section 3.1, we consider a test case, for which we compare the performances of our three strategies in terms of stabilization; in Section 3.2, we apply our strategies to the problem of achieving consensus for a multi-agent model: the Hegselmann-Krause bounded confidence model.

\section{Proof of Theorem 1.1}

In this section, we first recall several useful concepts used in the rest of the article. Given $\Omega \subset \mathbb{R}^{n}$ and $x \in \mathbb{R}^{n}$, we denote by $d(x, \Omega)=\min _{y \in \Omega}\|x-y\|$ the distance of $x$ to $\Omega$, and by $B_{\varepsilon}(\Omega)=\bigcup_{x \in \Omega} B_{\varepsilon}(x)=$ $\left\{y \in \mathbb{R}^{n} \mid d(y, \Omega)<\varepsilon\right\}$ the $\varepsilon$-neighborhood of $\Omega$. We now recall the definition of local attractiveness of a set $\Omega$ for a given dynamics. 
Definition 2.1. Consider the dynamics $\dot{x}=f(x)$, with $f$ Lipschitz on $\mathbb{R}^{n}$. We say that $\Omega$ is locally attractive for the dynamics if there exists $\varepsilon>0$ such that, for any $x_{0} \in B_{\varepsilon}(\Omega)$, the unique solution $x\left(t, x_{0}\right)$ of $\dot{x}=f(x)$ with initial data $x_{0}$ satisfies $d\left(x\left(t ; x_{0}\right), \Omega\right) \rightarrow 0$ as $t \rightarrow+\infty$.

We recall the definition of sampling solution, as introduced in [13], used in Strategy 2.

Definition 2.2 (Sampling solution). Let $U \subset \mathbb{R}^{m}, F: \mathbb{R}^{n} \times U \rightarrow \mathbb{R}^{n}$ be continuous and locally Lipschitz in $x$, uniformly on compact subsets of $\mathbb{R}^{n} \times U$. Given a feedback $u: \mathbb{R}^{n} \rightarrow U, \tau>0$, and $x_{0} \in \mathbb{R}^{n}$, we define the sampling solution of the Cauchy problem $\dot{x}=F(x, u(x)), x(0)=x_{0}$, as the continuous piecewise $C^{1}$ function $x:[0, T] \rightarrow \mathbb{R}^{n}$ solving recursively for $k \geq 0$

$$
\dot{x}(t)=F(x(t), u(x(k \tau))), \quad t \in[k \tau,(k+1) \tau]
$$

using as initial value $x(k \tau)$, the endpoint of the solution on the preceding interval, and starting with $x(0)=x_{0}$. We call $\tau$ the sampling time.

Finally we recall the definition of the $\omega$-limit of a trajectory.

Definition 2.3. Let $x(\cdot):[0,+\infty) \rightarrow \mathbb{R}^{n}$ be a curve. Its $\omega$-limit, that we denote by $\omega(x(\cdot))$, is the set of points $x_{*}$ such that there exists an increasing sequence $t_{n} \rightarrow+\infty$ such that $\lim _{n \rightarrow+\infty} x\left(t_{n}\right)=x_{*}$.

Note that, in our proofs, all trajectories are bounded, as a consequence of the fact that $V$ is a proper function and that $t \mapsto V(x(t))$ is nonincreasing (or, at worst, only slightly increasing on small time intervals in Strategy 2). As a consequence, all trajectories admit a nonempty $\omega$-limit.

\subsection{Strategy 1: Sparse time-varying periodic feedback}

In this section we prove point $(i)$ of Theorem 1.1. For every initial state $x_{0} \in \mathbb{R}^{n}$ and for every sampling time $\tau>0$, the control is well-defined and so is the corresponding trajectory of (1).

Proposition 2.1. Assume that $\Omega$ is locally attractive. For every initial state $x_{0} \in \mathbb{R}^{n}$, there exist $\tau_{1}>0$ and $T>0$ such that, for every $\tau \in\left(0, \tau_{1}\right)$, Strategy 1 with sampling time $\tau$ and final control time $T$ asymptotically stabilizes the control system (1) to $\Omega$.

Proof. Since $\Omega$ is locally attractive, there exists $\varepsilon>0$ such that any trajectory of $\dot{x}=f(x)$ with initial state in $B_{\varepsilon}(\Omega)$ converges to $\Omega$. Applying the classical Jurdjevic-Quinn theorem to (1) with the Control Lyapunov Function $\frac{1}{m} V$, the solution $y(\cdot)$ of

$$
\dot{y}=f(y)-\sum_{i=1}^{m} \frac{1}{m} L_{g_{i}} V(y) g_{i}(y), \quad y(0)=x_{0},
$$

converges asymptotically to $\Omega$. Let $T>0$ be such that $y(t) \in B_{\varepsilon / 2}(\Omega)$ for every $t \geq T$.

Denote by $x(t)$ the trajectory of (1) starting at $x_{0}$ associated with the control $u$ given by Strategy 1 with a given $\tau>0$ and the final time $T$ defined above.

Note that, since $L_{f} V(x)-\left(L_{g_{i}} V(x)\right)^{2} \leq 0$ for every $i=1, \ldots, m$ and for every $x \in \mathbb{R}^{N}$ then, for every $\tau>0$, the trajectory $x(\cdot)$ is contained in the compact set $V^{-1}\left(\left(-\infty, V\left(x_{0}\right)\right]\right)$ for all $t \geq 0$. Similarly, the trajectory $y(\cdot)$ of $(10)$ is contained in $V^{-1}\left(\left(-\infty, V\left(x_{0}\right)\right]\right)$ for all $t \geq 0$.

The control given by Strategy 1 can be written as

$$
u(t, x)=-\sum_{i=1}^{m} \alpha_{i}(t) L_{g_{i}} V(x),
$$


where $\alpha_{i}(t)$ is the $\tau m$-periodic function defined on $[0, \tau m)$ by

$$
\alpha_{i}(t)= \begin{cases}1 & \text { if } t \in[\tau(i-1), \tau i) \\ 0 & \text { otherwise }\end{cases}
$$

With this notation it is easy to see that (10) is the averaging of equation (1) with Strategy 1, namely

$$
f(x)-\sum_{i=1}^{m} \frac{1}{m} L_{g_{i}} V(x) g_{i}(x)=\frac{1}{\tau m} \int_{0}^{\tau m}\left(f(x)-\sum_{i=1}^{m} \alpha_{i}(t) L_{g_{i}} V(x) g_{i}(x)\right) d t .
$$

Therefore, by classical first-order averaging results (see, e.g., [30, Theorem 2.8.1]), there exists $C>0$ such that $\|x(t)-y(t)\|<C \tau$, for every $t \geq 0$. In particular, if $\tau_{1} \leq \varepsilon /(2 C)$, since $y(T) \in B_{\varepsilon / 2}(\Omega)$, we have $x(T) \in B_{\varepsilon}(\Omega)$. For $t>T$, the uncontrolled dynamics is attractive and thus steers $x(\cdot)$ to $\Omega$.

Consider now the whole compact set of initial states $x_{0} \in K$. By a compactness argument, there exists a $T>0$ such that all trajectories $y(t)$ of $(10)$ satisfy $y(T) \in B_{\varepsilon}(\Omega)$. By uniform compacness of all trajectories $x(\cdot)$ and $y(\cdot)$ starting from $K$, one can then find a uniform $C$ such that $\|x(t)-y(t)\|<C \tau$, for every $t \geq 0$, thus a uniform $\tau_{1}$.

The following example shows that the condition of local attractivity of $\Omega$ cannot be removed in Theorem 1.1 for Strategy 1.

Example 2.1. Consider a planar system, represented in polar coordinates $x=(r, \theta)$. Define the vector field $f(r, \theta)=\partial_{\theta}$ and two control vector fields $g_{1}, g_{2}$ satisfying the following property: for each $n \in \mathbb{N}$ it holds $g_{1}\left(2^{-n}, \theta\right)=2^{-n} \min \left\{0, \sin \left(2^{n} \theta\right)\right\} \partial_{r}$ and $g_{2}\left(2^{-n}, \theta\right)=2^{-n} \min \left\{0, \sin \left(2^{n} \theta+\pi\right)\right\} \partial_{r}$. Then extend $g_{1}$ and $g_{2}$ on $\mathbb{R}^{2}$ by defining $g_{1}=\phi_{1}(r, \theta) \partial_{r}$ and $g_{2}=\phi_{2}(r, \theta) \partial_{r}$ for two functions $\phi_{1}, \phi_{2}$ strictly negative for $r \neq 2^{-n}$. Such an extension is possible, because the values of $g_{1}, g_{2}$ converge to zero with bounded decay when $r$ tends to zero.

Consider the Lyapunov function $V=r^{2}$ and notice that $L_{f} V(r, \theta)=0$ for all $(r, \theta)$ and $L_{g_{1}} V(r, \theta)=$ $L_{g_{2}} V(r, \theta)=0$ for all points of the form $\left(2^{-n}, k 2^{-n} \pi\right)$ with $k=1, \ldots, 2^{1-n}$, and in $(0,0)$. Observe, moreover, that no point of the form $\left(2^{-n}, k 2^{-n} \pi\right)$ is invariant for the system, since $\dot{x}=f(x)$ steers it to $\left(2^{-n}, k 2^{-n} \pi+t\right)$ at time $t>0$. As a consequence, we have $\Omega=\{(0,0)\}$.

Strategy 1 is open-loop, i.e., the control does not depend on the actual state $x(t)$. Hence, the corresponding trajectories are solutions of the time-dependent dynamical system

$$
\dot{x}=f(x)-L_{g_{i}} V(x) g_{i}(x) \quad \text { for } t \in[(k m+i) \tau,(k m+i+1) \tau) .
$$

For this system, we have existence and uniqueness of the trajectory from a given point, forward and backward in time.

We now apply Strategy 1 from $x_{0}=(1,0)$ with $\tau=2^{-n}$, and we show that, for each $n \in \mathbb{N}$, the strategy does not steer $x_{0}$ to $\Omega$. By definition of $f$, and since both $g_{1}$ and $g_{2}$ have no component in the direction $\partial_{\theta}$, the strategy satisfies $x_{\tau}(t)=\left(r_{\tau}(t), t \bmod 2 \pi\right)$ for some function $r_{\tau}(t)$.

Consider also the trajectory $y_{\tau}(t)$ with $\tau=2^{-n}$ starting from $y_{0}=\left(2^{-n}, 0\right)$. On the time interval $\left[0,2^{-n}\right)$ the active vector field is $g_{1}$, that is always zero, and the trajectory is then $y_{\tau}(t)=$ $\left(2^{-n}, t \bmod 2 \pi\right)$; on the next time interval $\left[2^{-n}, 2 \cdot 2^{-n}\right)$ the active vector field is $g_{2}$, that is always zero, and the trajectory is $y_{\tau}(t)=\left(2^{-n}, t \bmod 2 \pi\right)$. This holds for the whole time interval $[0,2 \pi]$, and we have $y_{\tau}(2 \pi)=y_{\tau}(0)$, i.e., $y_{\tau}(\cdot)$ is a periodic trajectory, not converging to $\Omega$.

Assume now, by contradiction, that $x_{\tau}(t)=\left(r_{\tau}(t), t \bmod 2 \pi\right)$ converges to $\Omega$. In particular this implies that $r_{\tau}(t)$ tends to 0 , and that there exists $\bar{t}$ such that $r_{\tau}(\bar{t})=2^{-n}$. For such a $\bar{t}$, we have $x_{\tau}(\bar{t})=\left(2^{-n}, \bar{t} \bmod 2 \pi\right)=y_{\tau}(\bar{t})$. This contradicts uniqueness of the solution of (11). 
We have proved that, for each $\tau=2^{-n}$, Strategy 1 does not steer $x_{0}=(1,0)$ to $\Omega$. Since the sequence $2^{-n}$ tends to zero, for $x_{0}$ there exists no $\tau_{1}$ satisfying the conclusion of Proposition 2.1.

The following example shows that, in general, one cannot achieve practical stabilization (see e.g. $[32,24])$ with the Jurdjevic-Quinn method. This fact is not related to the sparsity constraint since the system below is single-input.

Example 2.2. Consider the one-dimensional system $\dot{x}=u\left(1-x^{2}\right)$ with the Lyapunov function $V=(x-1)^{2}$. It holds $f=0$ and $g=\left(1-x^{2}\right)$, hence $L_{f} V=0$ and the set $\mathcal{Z}=\Omega$ is reduced to two points $\{-1,+1\}$. By applying the Jurdjevic-Quinn Theorem, one chooses $u=-L_{g} V=-2(x-1)\left(1-x^{2}\right)$, hence the feedback system reads as

$$
\dot{x}=-2(x-1)\left(1-x^{2}\right)^{2} .
$$

Each trajectory starting at $x_{0} \leq-1$ converges to -1 , while each trajectory starting at $x_{0}>-1$ converges to 1 . Thus, by uniqueness of the trajectory, for each $\varepsilon>0$, the time $T$ such that $x(t) \in B_{\varepsilon}(\Omega)$ for all $t \geq T$ starting from $-1+\delta$ grows to infinity for $\delta \rightarrow 0$.

As a consequence, for the compact set $K=[-2,2]$ and for each sufficiently small $\varepsilon>0$ there exists no uniform $T>0$ such that each trajectory of (12) with initial point $x_{0} \in K$ satisfies $x(t) \in B_{\varepsilon}(\Omega)$ for all $t>T$.

\subsection{Strategy 2: Sparse sampled feedback}

Proposition 2.2. Assume that

$$
\left\{x \in \mathbb{R}^{n} \mid L_{f} V(x)=0 \text { and } L_{g_{i}} V(x)=0, \text { for } i=1, \ldots, m\right\}=\{\bar{x}\} .
$$

For every $x_{0} \in \mathbb{R}^{n}$, there exists a sampling time $\tau_{0}>0$ such that the sampling solution of (1) associated with $x_{0}, \tau<\tau_{0}$ and Strategy 2 asymptotically stabilizes the system to $\bar{x}$ in the sample-and-hold sense.

Proof. If $x_{0}=\bar{x}$, the trajectory reduces to $\bar{x}$ and there is nothing to prove. Fix $r>0$ and consider an initial condition $x_{0} \neq \bar{x}$. Choose $\rho>V\left(x_{0}\right)$ be such that the set $K=V^{-1}((-\infty, \rho])$, which is compact by assumption, contains both $B_{r}\left(x_{0}\right)$ and $B_{r}(\bar{x})$. The function $V$ has a minimum in the interior of $K$, which is realized in $\bar{x}$. Without loss of generality, we assume for simplicity that $\bar{x}=0$ and that $V(0)=0$. We are going to prove that there exist $\tau_{0}$ and $T$ such that the sampling trajectory with sampling time $\tau<\tau_{0}$ satisfies $|x(t)|<r$, for every $t \geq T$. This implies, in particular, the existence of $C$ such that $|x(t)| \leq C$ for every $t \geq 0$.

Let $L, M, \nu$ be positive constants such that

$$
\begin{array}{r}
|V(x)-V(y)| \leq L|x-y|, \quad\left|f(x)-L_{g_{i}} V(x) g_{i}(x)\right| \leq M \\
\left|L_{f} L_{g_{i}} V(x)\right|+\sum_{j=1}^{m}\left|L_{g_{j}} V(x)\right|\left|L_{g_{j}} L_{g_{i}} V(x)\right| \leq \nu, \\
\left|L_{f} L_{f} V(x)\right|+\sum_{j=1}^{m}\left|L_{g_{j}} V(x)\right|\left|L_{g_{j}} L_{f} V(x)\right| \leq \nu,
\end{array}
$$

for all $(x, y) \in K^{2}$ and $i \in\{1, \ldots, m\}$. Let $\varepsilon>0$ be such that $V^{-1}([0, \varepsilon)) \subset B_{r}(0)$, and define

$$
\mu=\min _{x \in K \backslash V^{-1}([0, \varepsilon / 2))} \max \left\{\left|L_{f} V(x)\right|,\left|L_{g_{1}} V(x)\right|, \ldots,\left|L_{g_{m}} V(x)\right|\right\} .
$$


Note that $\mu>0$ by (13). We set

$$
\tau_{0}=\min \left(\frac{\varepsilon}{2 L M}, \frac{\rho-V\left(x_{0}\right)}{L M}, \frac{\mu}{2 \nu}, \frac{1}{4 \nu}\right) .
$$

For any $\tau<\tau_{0}$ and for any $y \in V^{-1}\left(\left[0, x_{0}\right]\right)$, consider the sampling solution $x(\cdot)$ for $t \in[0, \tau]$ associated with $y, \tau$ and Strategy 2, i.e., the solution of

$$
\dot{x}=f(x)-L_{g_{i}} V(y) g_{i}(x), \quad x(0)=y,
$$

where the index $i \in\{1, \ldots, m\}$ is given by (3)-(4).

First, let us prove that for every $y \in V^{-1}\left(\left[0, x_{0}\right]\right)$ the solution $x(t)$ of $(15)$ remains in $K$ for every $t \in[0, \tau]$. By contradiction assume that $x(t)$ exits $K$ within time $\tau$. Then there exists a minimal $\bar{t} \in(0, \tau)$ such that $x(\bar{t}) \in \partial K$, i.e. $V(x(\bar{t}))=\rho$. In particular $x(t) \in K$ for every $t \in[0, \bar{t}$. Hence (14) holds true, and for every $t \in[0, \bar{t}]$ we have $V(x(t)) \leq V(y)+\tau L M \leq V\left(x_{0}\right)+\tau L M<\rho$, which raises a contradiction.

We now consider two cases: either $V(y)<\varepsilon / 2$ or $V(y) \geq \varepsilon / 2$.

Case 1. If $V(y)<\varepsilon / 2$ then $|V(x(t))-V(y)| \leq L|x(t)-y| \leq t L M \leq \tau_{0} L M<\frac{\varepsilon}{2}$ for every $t \in[0, \tau]$. In particular the solution starting at $y$ remains in $V^{-1}([0, \varepsilon))$ in a single sampling step.

Case 2. If $V(y) \geq \varepsilon / 2$, we have

$$
\begin{aligned}
\left|L_{g_{i}} V(x(t))-L_{g_{i}} V(y)\right| & \leq t \sup _{t \in[0, \tau]}\left|\frac{d}{d t} L_{g_{i}} V(x(t))\right| \\
& \leq \tau \sup _{x \in K}\left(\left|L_{f} L_{g_{i}} V(x)\right|+\sum_{j=1}^{m}\left|L_{g_{j}} V(x)\right|\left|L_{g_{j}} L_{g_{i}} V(x)\right|\right) \leq \tau \nu .
\end{aligned}
$$

Similarly, we have

$$
\left|L_{f} V(x(t))-L_{f} V(y)\right| \leq \tau \nu
$$

We have two sub-cases:

Case 2.1. If $\left|L_{g_{j}} V(y)\right|<\mu$ for every $j=1, \ldots, m$, then (17) implies $L_{f} V(y) \leq-\mu$. The estimates (17) gives that $L_{f} V(x(t)) \leq L_{f} V(\mu)+\tau \nu<-\mu / 2$. Let $i \in\{1, \ldots, m\}$ be the index given by (3) in Strategy 2. If $L_{g_{i}} V(x(t)) L_{g_{i}} V(y) \geq 0$ for all $t \in[0, \tau]$ then

$$
\frac{d}{d t} V(x(t))=L_{f} V(x(t))-L_{g_{i}} V(y) L_{g_{i}} V(x(t))<-\frac{\mu}{2}, \quad \text { for every } t \in[0, \tau] .
$$

Otherwise if $L_{g_{i}} V(y) L_{g_{i}} V(x(t))<0$ for some $t \in[0, \tau]$ then let $\bar{t} \in[0, \tau]$ such that $L_{g_{i}} V(x(\bar{t}))=0$. Then, following (16), one has $\left|L_{g_{i}} V(x(t))\right|=\left|L_{g_{i}} V(x(t))-L_{g_{i}} V(x(\bar{t}))\right| \leq(\tau-\bar{t}) \nu<1 / 4$ since $\tau<\frac{1}{4 \nu}$. Hence $L_{g_{i}} V(x(t)) L_{g_{i}} V(y)>-\frac{\mu}{4}$, which gives

$$
\frac{d}{d t} V(x(t))=L_{f} V(x(t))-L_{g_{i}} V(y) L_{g_{i}} V(x(t))<-\frac{\mu}{2}+\frac{\mu}{4}=-\frac{\mu}{4}, \quad \text { for every } t \in[0, \tau] .
$$

Case 2.2. If $\left|L_{g_{j}} V(y)\right| \geq \mu$ for some $j=1, \ldots, m$, let $i \in\{1, \ldots, m\}$ be the index given by (3) in Strategy 2. In particular $\left|L_{g_{i}} V(y)\right| \geq \mu$. Then, from (16) we have that $\left|L_{g_{i}} V(x(t))-L_{g_{i}} V(y)\right|<\frac{\mu}{2}$ and hence $L_{g_{i}} V(x(t)) L_{g_{i}} V(y)>\frac{\mu^{2}}{2}$ for every $t \in[0, \tau]$. This implies

$$
\frac{d}{d t} V(x(t))=L_{f} V(x(t))-L_{g_{i}} V(y) L_{g_{i}} V(x(t))<-\frac{\mu^{2}}{2}, \quad \text { for every } t \in[0, \tau] .
$$


Summing up Case 2: if $V(y) \geq \frac{\varepsilon}{2}$, then the functional $V$ is strictly decreasing for all times $t \in[0, \tau]$ at a rate smaller than $\max \left\{-\mu / 4,-\mu^{2} / 2\right\}<0$.

Finally, summing up Case 1 and Case 2: if we consider the sampling solution $x(t)$ of (1) associated with an initial condition $x_{0} \in V^{-1}([0, \varepsilon / 2)), \tau<\tau_{0}$ and the control defined by (3)-(4), we have $x(t) \in V^{-1}([0, \varepsilon))$ for every $t \geq 0$. If $x_{0} \notin V^{-1}([0, \varepsilon / 2))$ then while $x(t) \notin V^{-1}([0, \varepsilon / 2))$ the Lyapunov function is strictly decreasing and in particular $V(x(\tau))<V\left(x_{0}\right)$. By recurrence, the solution stays in $V\left(\left[0, x_{0}\right]\right)$ for every $t \in[0, \tau]$. Moreover, while $x(t) \notin V^{-1}([0, \varepsilon / 2))$ the decay rate of the Lyapunov function is bounded by $\max \left\{-\mu / 4,-\mu^{2} / 2\right\}<0$ and the trajectory reaches the sublevel $V^{-1}([0, \varepsilon / 2))$ in finite time. Then (see Case 1$) x(t) \in V^{-1}([0, \varepsilon))$ for every $t \geq T$.

Remark 2.1. The main difference between Strategy 2 and Strategies 1 or 3 is that, in general, Strategy 2 does not provide stability whenever $\mathcal{Z}=\left\{x \in \mathbb{R}^{n} \mid L_{f} V(x)=0\right.$ and $L_{g_{i}} V(x)=0$, for $\left.i=1, \ldots, m\right\}$ is not reduced to a single point. In Strategy 2, unlike the other strategies, the control is defined by evaluating the Lie derivatives $L_{g_{i}} V(x(t))$ on discrete times $k \tau$ only. This fact represents an advantage from the computational point of view but, on the other hand, makes the strategy less robust.

In particular, when the trajectory is close to $\mathcal{Z}$ the value of the Lyapunov function $V$ may be slightly increasing. Then, if $\mathcal{Z}$ is not reduced to a point, Strategy 2 may define a trajectory staying close to $\mathcal{Z}$ for a large interval of time producing a non-negligible increase of $V$, then leaving a neighborhood of $\mathcal{Z}$ (see Figure 1). Moreover, when $\mathcal{Z}$ is an unbounded manifold, Strategy 2 may even define a strategy staying close to $\mathcal{Z}$ but escaping every compact.

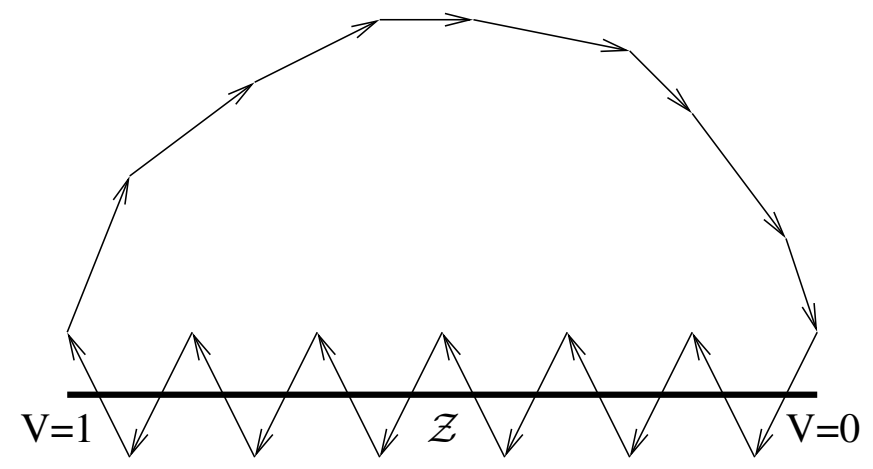

Figure 1: Strategy 2 may fail to stabilize in the sample-and-hold sense the system to $\mathcal{Z}$ if $\mathcal{Z}$ is not reduced to a point.

\subsection{Strategy 3: Sparse feedback with hysteresis}

Proposition 2.3. Let $\varepsilon \in(0,1)$ be fixed. For every $x_{0} \in \mathbb{R}^{n}$, Strategy 3 asymptotically stabilizes the control system (1) to $\Omega$.

Proof. The proof goes in three steps: we first prove that the control strategy is well-defined, by proving that the sequence of switching times $t_{n}$ is well-defined and it cannot converge to a finite value. We then prove that the corresponding trajectory converges to the set $\mathcal{Z}$, and finally prove that it converges to $\Omega \subset \mathcal{Z}$.

Step 1. We first prove that the trajectory associated with the hysteresis is well defined for every $t \geq 0$. First note that, when a control $u$ is chosen at time $t_{n}$, there exists $\eta>0$ such that the control is defined on $\left[t_{n}, t_{n}+\eta\right)$. In particular, the next switching time $t_{n+1}$ satisfies $t_{n+1}>t_{n}$, if it exists. 
We prove that the sequence of times $t_{n}$ is well defined, by iteration. Note that, for $t_{0}=0$, we have $\left|L_{g_{i}} V\left(x\left(t_{0}\right)\right)\right|<2 t_{0}^{-1}=+\infty$, hence there exists a unique index $i$ satisfying condition (5). By continuity of the function $\left|L_{g_{i}} V(x(t))\right|$ with respect to $t$, either there exists $t_{1}>t_{0}$ for which $\left|L_{g_{i}} V\left(x\left(t_{1}\right)\right)\right| \geq 4 t_{1}^{-1}$ or it holds $t_{1}=+\infty$ in the case in which $\left|L_{g_{i}} V(x(t))\right|<4 t^{-1}$ for all times $t \geq t_{0}$.

Assume that $t_{n}$ is well defined. Then, there exists a unique index $i$ satisfying (5). If $\left|L_{g_{i}} V\left(x\left(t_{n}\right)\right)\right|<$ $2 t_{n}^{-1}$, then the existence of $t_{n+1}$ is equivalent to the existence of $t_{1}$ starting from $t_{0}$. If $\left|L_{g_{i}} V\left(x\left(t_{n}\right)\right)\right| \geq$ $2 t_{n}^{-1}$, then there exists at most one minimal $\hat{t}>t_{n}$ solving $\left|L_{g_{i}} V(x(t))\right| \leq t^{-1}$. Similarly, since $\left|L_{g_{i}} V\left(x\left(t_{n}\right)\right)\right||\geq| L_{g_{j}} V\left(x\left(t_{n}\right)\right) \mid$ for all $j \in\{1, \ldots, m\}$, then there exists at most one minimal $\bar{t}>t_{n}$ solving $\left|L_{g_{i}} V(x(\bar{t}))\right| \leq(1-\varepsilon)\left|L_{g_{j}} V(x(\bar{t}))\right|$ for some $j \in\{1, \ldots, m\}$. Then $t_{n+1}=\min \{\hat{t}, \bar{t}\}$, with the convention that $t_{n+1}=+\infty$ if both $\hat{t}, \bar{t}$ are undefined. Therefore $t_{n+1}$ is well defined.

We now prove that the sequence $\left(t_{n}\right)_{n \in \mathbb{N}}$ cannot converge to a finite value. First note that a limit exists, since $\left(t_{n}\right)_{n \in \mathbb{N}}$ is increasing. Assume by contradiction that $\lim _{n \rightarrow+\infty} t_{n}=T<+\infty$. Since the strategy is well defined on all time intervals $\left[t_{n}, t_{n+1}\right) n \geq 0$, then the trajectory $x(\cdot)$ is well-defined on the whole interval $[0, T)$. Note that the function $V(x(\cdot))$ is nonincreasing since $\dot{V}=L_{f} V \leq 0$ or $\dot{V}=L_{f} V-\left|L_{g_{i}} V\right|^{2} \leq 0$. Then $x(t) \in V^{-1}((-\infty, V(x(0))])$ for every $t \in[0, T)$. Moreover, the trajectory is Lipschitz with respect to time, hence there exists a limit $x^{*}=\lim _{t \rightarrow T} x(t)$. Let $C=\max _{i=1, \ldots, m}\left|L_{g_{i}} V\left(x^{*}\right)\right|$. We now have three cases:

1. If $C<2 T^{-1}$ then, by continuity, there exists $\bar{t}$ such that $\left|L_{g_{i}} V(x(t))\right|<2 t^{-1}$ for all $t \in[\bar{t}, T)$ and $i=1, \ldots, m$. Let $n$ be sufficiently large so that $t_{n} \in[\bar{t}, T)$. Consider the corresponding index $i$ satisfying condition (5). Then $\left|L_{g_{i}} V\left(x\left(t_{n}\right)\right)\right|<2 t_{n}^{-1}$, and for every $t \in\left[t_{n}, T\right)$ one has $\left|L_{g_{i}} V(x(t))\right| \leq 4 t^{-1}$. Then, $t_{n+1} \geq T$, hence $t_{n+2}>T$ and $T$ is not the limit of $\left(t_{n}\right)_{n \in \mathbb{N}}$.

2. If $C>2 T^{-1}$, then there exists $\bar{t}$ such that $\max _{i=1, \ldots, m}\left|L_{g_{i}} V(x(t))\right|>2 t^{-1}$ for all $t \in[\bar{t}, T)$. Split $\{1, \ldots, m\}$ into the sets $I=\left\{i\right.$ s.t. $\left.\left|L_{g_{i}} V\left(x^{*}\right)\right|=C\right\}$ and $J=\left\{i\right.$ s.t. $\left.\left|L_{g_{i}} V\left(x^{*}\right)\right|<C\right\}$. Then, for each sufficiently small $\eta>0$, there exists a time $\hat{t} \in[\bar{t}, T)$ such that for all times $t \in[\hat{t}, T)$ and $i \in I$ we have $\left|L_{g_{i}} V(t)\right| \in[C-\eta, C+\eta]$, and for all times $t \in[\hat{t}, T)$ and $i \in J$ we have $\left|L_{g_{i}} V(t)\right| \leq C-2 \eta$. Reducing $\eta$ if necessary, we assume that $C-\eta>(1-\varepsilon)(C+\eta)$ without loss of generality. Choose $n$ sufficiently large to have $t_{n} \in[\hat{t}, T)$, and consider the corresponding index $i$ satisfying condition (5), which belongs to $I$. Then $\left|L_{g_{i}} V\left(x\left(t_{n}\right)\right)\right|>2 t_{n}^{-1}$ and the switching time $t_{n+1} \notin\left[t_{n}, T\right)$, since we have both $\left|L_{g_{i}} V(x(t))\right|>t^{-1}$ and $\left|L_{g_{i}} V(x(t))\right| \geq C-\eta>(1-\varepsilon)(C+\eta) \geq$ $(1-\varepsilon)\left|L_{g_{j}} V(x(t))\right|$ for all $t \in\left[t_{n}, T\right)$ and $j \neq i$. Then, $t_{n+1} \geq T$, hence $T$ is not the limit of $\left(t_{n}\right)_{n \in \mathbb{N}}$.

3. If $C=2 T^{-1}$, then there exists $\bar{t}$ such that $\max _{i=1, \ldots, m}\left|L_{g_{i}} V(x(t))\right| \in\left(t^{-1}, 4 t^{-1}\right)$ for all $t \in[\bar{t}, T)$. As in Case 2, define the sets of indexes $I, J$, the constant $\eta>0$ and the time $\hat{t}$. Take now any $t_{n}$ in the interval $[\hat{t}, T)$ and consider the index $i$ satisfying (5) at time $t_{n}$, that belongs to the set $I$. If $\left|L_{g_{i}} V\left(x\left(t_{n}\right)\right)\right| \geq 2 t_{n}^{-1}$, then the switching condition (6) is never satisfied in $\left[t_{n}, T\right)$, for the same reasons as in Case 2. If $\left|L_{g_{i}} V\left(x\left(t_{n}\right)\right)\right|<2 t_{n}^{-1}$, then the switching condition (7) is never satisfied, since $\left|L_{g_{i}} V(x(t))\right|<4 t^{-1}$ by construction. In both cases, $t_{n+1} \geq T$ and $T$ is not the limit of $\left(t_{n}\right)_{n \in \mathbb{N}}$.

Summing up, $t_{n}$ cannot converge to a finite value $T$, hence either there exists $t_{n}=+\infty$ or $t_{n}$ goes to $+\infty$. In both cases, Strategy 3 is well defined on $[0,+\infty)$.

Step 2. We now prove that Strategy 3 steers $x(\cdot)$ to the set

$$
\mathcal{Z}=\left\{x \in \mathbb{R}^{n} \mid L_{f} V(x)=0, L_{g_{i}} V(x)=0, \text { for every } i=1, \ldots, m\right\} .
$$


First note that, by construction, $\dot{V}(x(t))=L_{f} V$ or $\dot{V}(x(t))=L_{f} V-\left|L_{g_{i}} V\right|^{2}$, hence $\dot{V}(x(t)) \leq 0$. Since $V$ is proper and smooth, then $V(x(\cdot))$ is bounded below. Then, $V(x(\cdot))$ admits a limit, hence $\lim _{t \rightarrow+\infty} \dot{V}(x(t))=0$ (similarly to Step 1 in the proof of Proposition 2.1). Since $\dot{V} \leq L_{f} V \leq 0$, we get $\lim _{t \rightarrow+\infty} L_{f} V(x(t))=0$, i.e., the $\omega$-limit of the trajectory $x(\cdot)$ satisfies $\omega(x(\cdot)) \subset\left\{L_{f} V=0\right\}$.

Consider now the sequence of switching times $t_{n}$ defined by Strategy 3. We study the function $L_{g_{i}} V(x(\cdot))$ on the interval $\left[t_{n}, t_{n+1}\right)$ We have two possibilities:

- If $\max _{i=1, \ldots, m}\left|L_{g_{i}} V\left(x\left(t_{n}\right)\right)\right|<2 t_{n}^{-1}$, then, by definition of the switching time $t_{n+1}$, we have $\left|L_{g_{j}} V(x(t))\right| \leq 4 t^{-1}$ for all $t \in\left[t_{n}, t_{n+1}\right)$ and $j=1, \ldots, m$.

- If $\max _{i=1, \ldots m}\left|L_{g_{i}} V\left(x\left(t_{n}\right)\right)\right| \geq 2 t_{n}^{-1}$, then, noting that $\dot{V}=L_{f} V-\left|L_{g_{i}} V\right|^{2}$ on such a time interval, and recalling that $L_{f} V \leq 0$, we have $\left|L_{g_{i}} V\right|^{2}=L_{f} V-\dot{V} \leq|\dot{V}|$. Recalling that $\left|L_{g_{i}} V(x(t))\right| \geq$ $(1-\varepsilon)\left|L_{g_{j}} V(x(t))\right|$ for all $t \in\left[t_{n}, t_{n+1}\right)$, we have $\left|L_{g_{j}} V(x(t))\right| \leq(1-\varepsilon)^{-1}|\dot{V}(x(t))|^{1 / 2}$ for all $t \in\left[t_{n}, t_{n+1}\right)$ and $j=1, \ldots, m$.

Summing up, we have $\left|L_{g_{j}} V(x(t))\right| \leq \max \left\{4 t^{-1},(1-\varepsilon)^{-1} \sqrt{|\dot{V}(x(t))|}\right\}$ for all $t \in[0,+\infty)$ and $j=$ $1, \ldots, m$. Since both $4 t^{-1}$ and $(1-\varepsilon)^{-1} \sqrt{|\dot{V}(x(t))|}$ converge to zero, we have $\lim _{t \rightarrow+\infty} L_{g_{j}} V(x(t))=0$, i.e. $\omega(x(\cdot)) \subset\left\{L_{g_{j}} V=0\right\}$ for all $j=1, \ldots, n$. Then $\omega(x(\cdot)) \subset \mathcal{Z}$.

Step 3. We finally prove that Strategy 3 steers the system to the set $\Omega$ being the largest invariant subset of $\mathcal{Z}$ under the uncontrolled dynamics $\dot{x}=f(x)$. The difficulty is to prove that the limit set of the controlled trajectory is in an invariant set under the uncontrolled dynamics.

Consider the trajectory $x(\cdot)$ given by Strategy 3, and $x_{*}$ belonging to the $\omega$-limit of $x(\cdot)$. By definition, there exists $\tau_{n} \rightarrow+\infty$ such that $\lim _{n \rightarrow+\infty} x\left(\tau_{n}\right)=x_{*}$. Fix $t \geq 0$ and consider on one side the sequence $x_{n}=x\left(\tau_{n}+t\right)$. On the other side, consider the point $y(t)$ being the unique solution at time $t$ of the Cauchy problem $\dot{y}=f(y), y(0)=x_{*}$. Let us prove that $\lim _{n \rightarrow+\infty} x_{n}=y(t)$. By definition of Strategy 3, we have the following estimate for $s \in[0, t]$ :

$$
\left|\dot{x}\left(\tau_{n}+s\right)-\dot{y}(s)\right| \leq \operatorname{Lip}(f)\left|x\left(\tau_{n}+s\right)-y(s)\right|+\left|L_{g_{i}} V\left(x\left(\tau_{n}+s\right)\right)\right|\left|g_{i}\left(x\left(\tau_{n}+s\right)\right)\right|,
$$

where $g_{i}$ is the active vector field for Strategy 3, if it exists. By compactness, both the Lipschitz constant of $f, \operatorname{Lip}(f)$, and the norm $\left|g_{i}\left(x\left(\tau_{n}+s\right)\right)\right|$ are bounded by some $M>0$. Then, Gronwall estimates give

$$
\left|x\left(\tau_{n}+t\right)-y(t)\right| \leq e^{M t}\left|x\left(\tau_{n}\right)-x_{*}\right|+\frac{e^{M t}-1}{M} M \int_{\tau_{n}}^{\tau_{n}+t}\left|L_{g_{i}} V(x(s))\right| d s .
$$

Note that $\lim _{n \rightarrow+\infty} x\left(\tau_{n}\right)=x_{*}$. Moreover, $\left|L_{g_{i}} V(x(s))\right| \rightarrow 0$ for $s \rightarrow+\infty$, hence the integral on an interval of fixed length tends to zero too. Since $t$ is fixed, this implies $\lim _{n \rightarrow+\infty} x\left(t_{n}+t\right)=y(t)$, hence $y(\cdot)$ is in the $\omega$-limit of the trajectory $x(\cdot)$. By Step 2 of the proof, this implies that $y(t) \in \mathcal{Z}$. Since $t$ is arbitrary, the whole trajectory $y(\cdot)$ belongs to $\mathcal{Z}$ and thus to the largest invariant subset of $\mathcal{Z}$ under the dynamics $\dot{y}=f(y)$. Then, $y(t) \in \Omega$ for any $t$, and in particular $y(0)=x_{*} \in \Omega$.

Remark 2.2. The three threshold time-dependent functions used in the definition of Strategy 3 satisfy $t^{-1}<2 t^{-1}<4 t^{-1}$. One can easily see that they can be replaced with three positive functions satisfying $\phi_{1}(t)<\phi_{2}(t)<\phi_{3}(t)$ converging to 0 as $t \rightarrow+\infty$. In particular, the functions can take finite values for $t=0$, by maybe allowing one control to be active along $\left[0, t_{1}\right]$. 


\section{Applications}

\subsection{A test case}

We test here our three strategies on the simple control system $\dot{x}=u, \dot{y}=v$ in $\mathbb{R}^{2}$, showing that they provide convergence to the origin with different speeds of convergence. We consider the Lyapunov function $V(x, y)=\frac{1}{2} x^{2}+\frac{1}{2} y^{2}$. The usual Jurdjevic-Quinn method provides the non-sparse feedback control $(u, v)=(-x,-y)$.

Theorem 1.1 can be obviously applied with $\mathcal{Z}=\Omega=\{(0,0)\}$. In order to evaluate the speed of convergence, let us compute the time required to reach the circle of radius 1 centered at the origin, from any initial state (by uniqueness of the solution the time needed to reach the origin is always infinite). By symmetry, we can restrict our analysis to the subset $\{x \geq 0, y \geq 0\}$.

Figures 2, 3, and 4 display the level sets of the function time-to-target for the sampling times $\tau \in\{1,0.1,0.01\}$, for Strategy 1,2 , and 3 respectively.

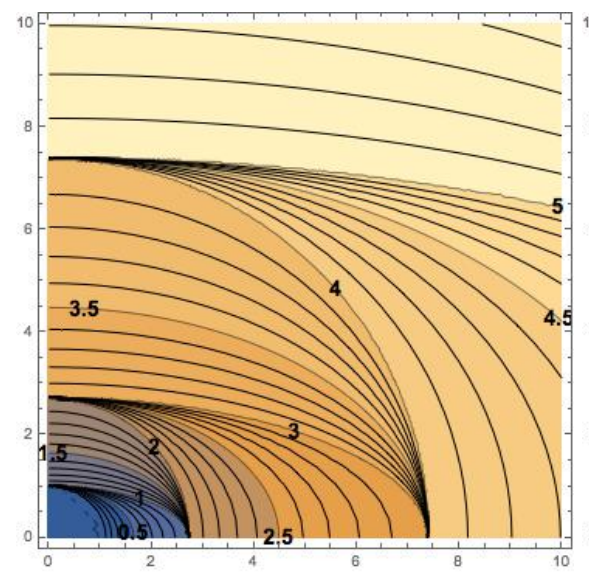

(a) $\tau=1$

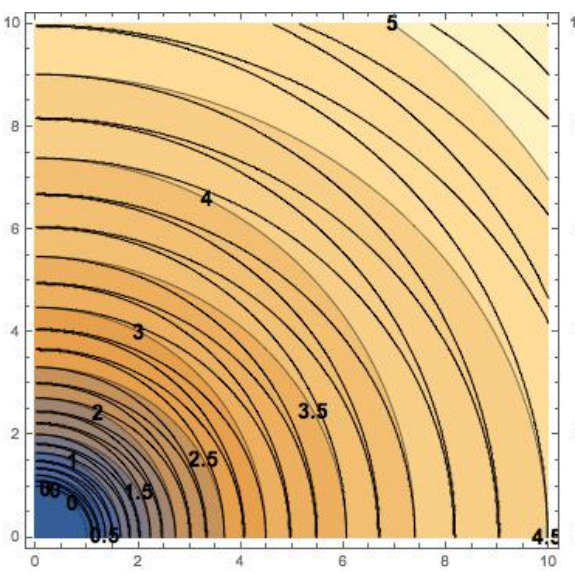

(b) $\tau=0.1$

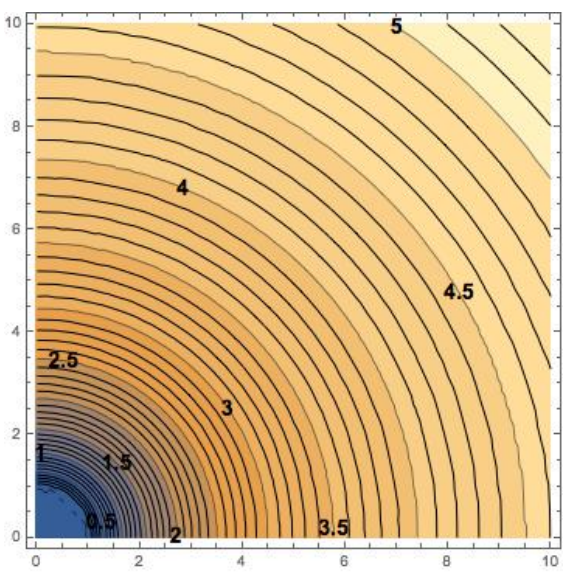

(c) $\tau=0.01$

Figure 2: Time to target for Strategy 1

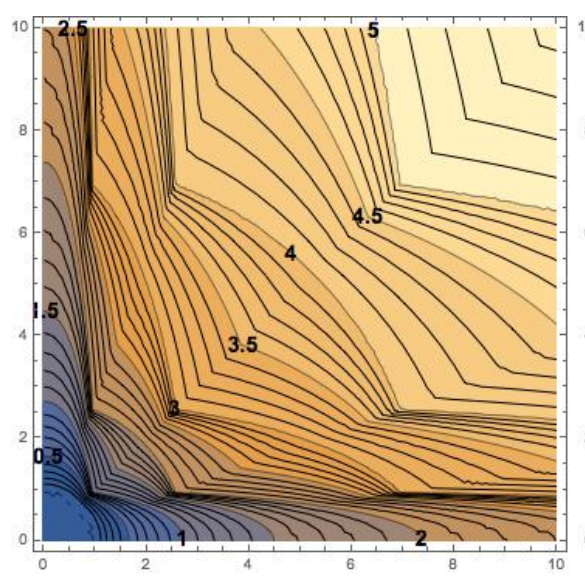

(a) $\tau=1$

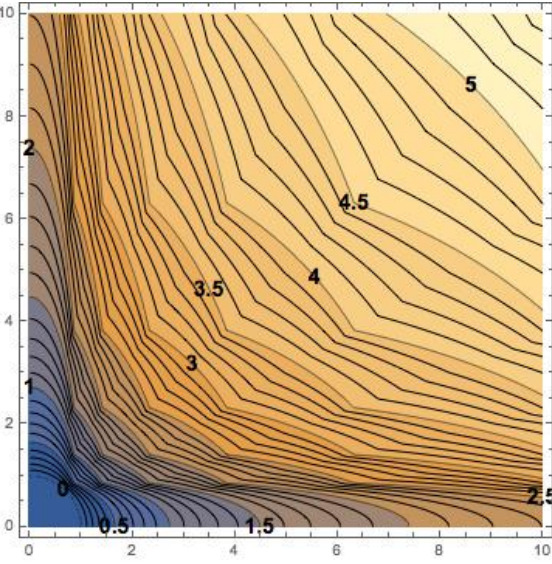

(b) $\tau=0.5$

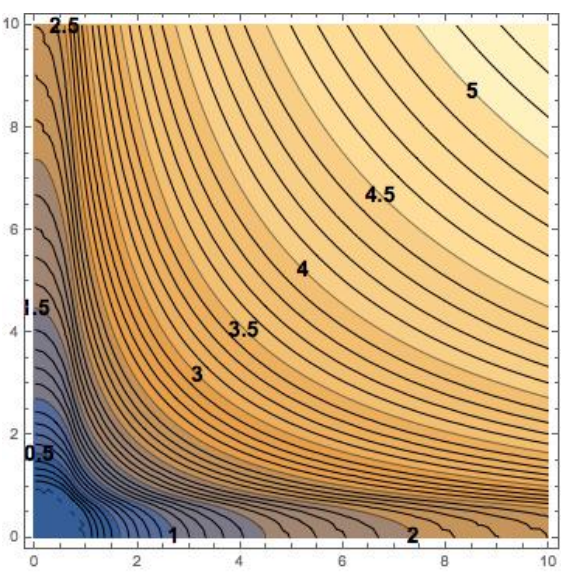

(c) $\tau=0.01$

Figure 3: Time to target for Strategy 2 


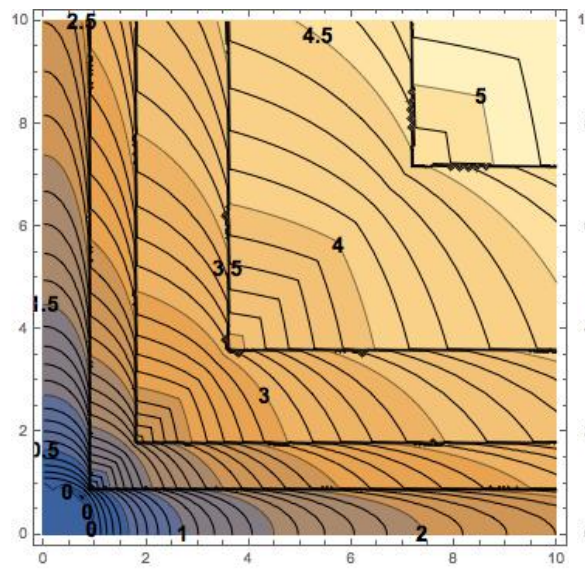

(a) $\varepsilon=0.5$

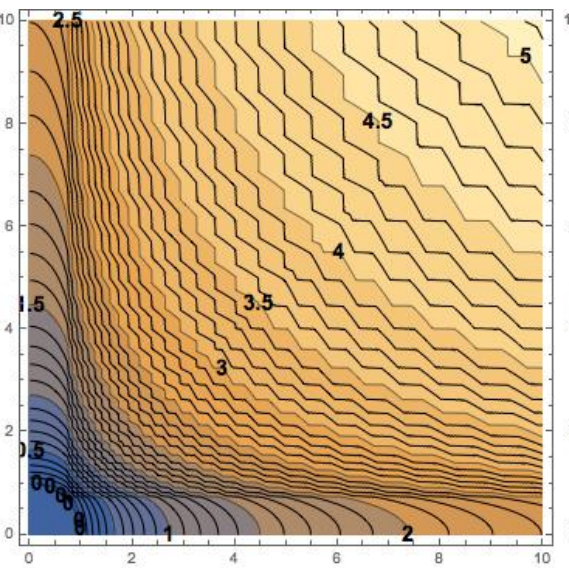

(b) $\varepsilon=0.1$

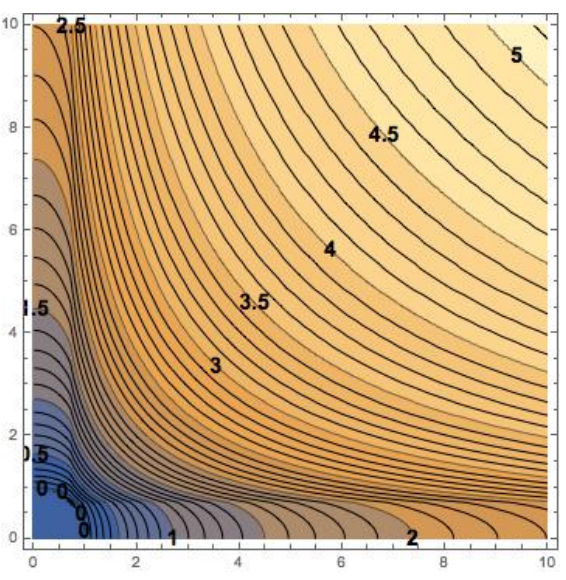

(c) $\varepsilon=0.01$

Figure 4: Time to target for Strategy 3

For Strategy 1, there is a strong discontinuity for large values of $\tau$. Indeed, for an initial data $\left(e^{\eta}, 0\right)$ with $\eta \in(0, \tau]$, Strategy 1 is reduced to one step: the sparse control $(u, v)=(-x, 0)$ on the time interval $[0, \eta)$ steers the initial data to the target in time $\eta$. If, otherwise, the initial data are $\left(e^{\eta}, 0\right)$ with $\eta \in(\tau, 2 \tau]$ Strategy 1 consists of three steps:

1. the sparse control $(u, v)=(-x, 0)$ on $[0, \tau)$ steers the initial data to $\left(e^{\eta-\tau}, 0\right)$;

2. the sparse control $(u, v)=(0,-y)$ on $[\tau, 2 \tau)$ keeps the state in $\left(e^{\eta-\tau}, 0\right)$;

3. the sparse control $(u, v)=(-x, 0)$ on $[2 \tau, \tau+\eta)$ steers the state $\left(e^{\eta-\tau}, 0\right)$ to the target.

The discontinuity is then of the order of $\tau$, hence the function is smooth for $\tau$ small. Moreover, the function time-to-target converges to a radial function, which coincides with the time to the target when the control is given by the standard Jurdjevic-Quinn method when replacing $V$ by $\frac{1}{2} V$.

The example also shows the main drawback of Strategy 1. The time-dependent approach, not taking into account the steepest descent for $V$, may introduce time intervals (such as Step 2 above) in which $V$ decreases very slowly or is constant, along which stabilization is not efficient.

For Strategy 2, the function time-to-target is Lipschitz continuous but not differentiable. The function is smooth for $\tau$ small.

It is interesting to notice that the limit of the control strategy does not exist, but that a limit function time-to-target does exist and it is regular. Indeed, the limit strategy consists of taking $(u, v)=(-x, 0)$ for $x>y$ and $(u, v)=(0,-y)$ for $x<y$. In both cases, this allows to converge to the manifold $\{x=y\}$. Along this manifold, chattering occurs and the limit strategy is not defined. This is the main motivation for using stabilization in the sample-and-hold sense in Definition 1.1. Nevertheless, the limit function time-to-target exists along the manifold $\{x=y\}$, and it coincides with the function time-to-target for the control $(u, v)=\left(-\frac{1}{2} x,-\frac{1}{2} y\right)$.

For Strategy 3, even though the function time-to-target is continuous, a remarkable change appears for $\varepsilon=0.5$ on the boundary of the subset $A=\{x>1, y>1\}$. This is due to the fact that, for points in $\{x \geq 0, y \geq 0\} \backslash A$, the target is reached with no switching, while for points in $A$, the controls have one or more switchings. Similarly, a careful look at the case $\varepsilon=0.1$ shows that the function 
time-to-target is highly irregular, in particular for points close to the manifold $\{x=y\}$, for which the number of switchings increases as $\varepsilon$ tends to 0 . Similarly to the previous case, the limit of the control strategy does not exist, but a limit function time-to-target does exist and it is regular. In particular, the limit function time-to-target for Strategies 2 and 3 coincide.

Finally we compare the limits of the three strategies. Figure 5 displays the subset for which the time to target is 2, for Strategy 1 (orange circle) and Strategies 2 and 3 (coinciding, red curve), respectively. This shows that Strategies 2 and 3 outperform Strategy 1, in particular for initial states near the axes. Two reasons explain such a result: first, for Strategy 1 control near axes is close to zero for half of times. More generally, the chosen control in Strategy 1 at a given time is not related to any optimality condition, such as the steepest descent for Strategies 2 and 3.

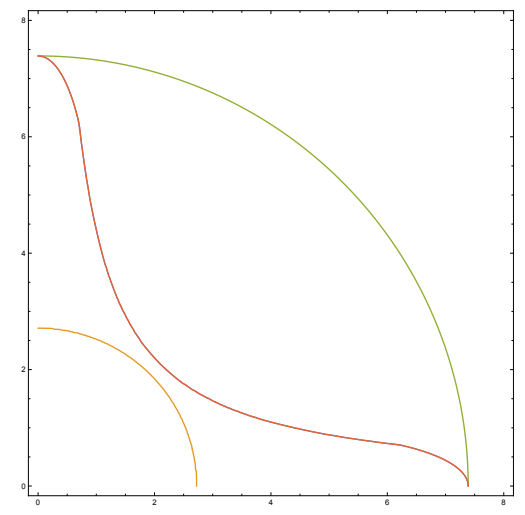

Figure 5: Set with time to target equal to 2 for limits of Strategy 1 (orange circle), Strategies 2 and 3 (coinciding, red curve) and non-sparse Jurdjevic-Quinn control (green circle).

On the same figure, we also compare the strategies with the control computed by the standard (non-sparse) Jurdjevic-Quinn method $(u, v)=(-x,-y)$. It clearly outperforms Strategies 2 and 3, in particular near the line $\{x=y\}$, in which the sparse strategies chatter.

\subsection{Consensus enforcement in multi-agent systems}

Multi-agent systems, with their self-organized emergent behaviors, provide a natural example of dissipative system. The intrinsic relation between dissipative systems and self-organization is a classical topic that dates back to the seminal works of Ilya Prigogine in thermodynamics (see for instance [26]). The analysis of self-organized behaviors, and more generally of multi-agent dynamics, has been the object of investigations in a number of situations, ranging from linguistics to distributed computing, to physics and animal behavior. In mathematics, multi-agent systems have attracted the attention of many researchers in the last decades (see for instance the survey [25]). Here, we focus on first-order consensus models, usually called opinion formation models. We prove that each of the three strategies presented in this article steers the system to global consensus, by acting only on at most one agent at any instant of time. The only controlled agent wears, in some sense, the role of instantaneous leader of the group.

Sparse stabilization and controllability for multi-agent models has been introduced in [9, 10] for alignment systems. The control strategy is based on a sampling technique analogous to Strategy 2. With the same method, in [5] a non-global sparse stabilization method was proved for a system submitted to repulsion and attraction forces (see [16]). Beside the sparse controllability, we mention 
also the controllability via leadership, which deals with single-input control-affine systems (or when $m \ll n$, see $[1,2,7,20,33,17]$.

We consider a first-order model for $N$ agents, represented by the vector of their positions $x \in \mathbb{R}^{N}$, interacting one with each other according to

$$
\dot{x}_{i}=\sum_{j \neq i} a_{i j}\left(x_{j}-x_{i}\right) \quad \text { for } i=1, \ldots, N
$$

for some interaction coefficients $a_{i j} \geq 0$. First-order consensus dynamics of this kind are often called opinion formation models, since they may model the evolution of the opinions $x_{i}$. For instance, one of the most influential models in opinion formation is the Bounded Confidence Model by Hegselmann and Krause [21] (see also [4]). The main feature of this model is that the interaction is zero when the distance between two opinions is larger than a certain threshold,

$$
a_{i j}= \begin{cases}1 & \text { if }\left|x_{i}-x_{j}\right| \leq 1 \\ 0 & \text { otherwise }\end{cases}
$$

It has been proved in [4] that for almost all initial configurations ${ }^{1}$, the opinions converge asymptotically to clusters. In particular, the system does not in general reach global consensus.

Here, we consider the more general controlled first-order consensus model

$$
\dot{x}_{i}=\sum_{j \neq i} \phi\left(x_{j}-x_{i}\right)\left(x_{j}-x_{i}\right)+u_{i} \quad \text { for } i=1, \ldots, N
$$

where the function $\phi$ is defined by

$$
\phi(x)= \begin{cases}1 & \text { if }|x|<1 \\ -\frac{|x|}{\eta}+1+\frac{1}{\eta} & \text { if }|x| \in[1,1+\eta] \\ 0 & \text { if }|x|>1+\eta\end{cases}
$$

for some small $\eta>0$. This is a variant of the Hegselmann-Krause model, in which the Lipschitzianity of $\phi$ ensures existence and uniqueness of the solutions of (18). This system can be written in the form (1), with $f=\sum_{i=1}^{N} e_{i} \sum_{j \neq i} \phi\left(x_{j}-x_{i}\right)\left(x_{j}-x_{i}\right)$ and $g_{i}=e_{i}$, where $e_{i}$ is the unit vector in the $i$-th variable.

It is well known that, for such a dynamical system, the variance functional

$$
V=\sum_{i, j=1, \ldots, N}\left(x_{i}-x_{j}\right)^{2}
$$

satisfies $L_{f} V \leq 0$. However, the functional $V$ is not proper, since it is equal to zero along the subspace $C=\left\{x_{1}=\cdots=x_{N}\right\}$, the so-called consensus manifold, i.e., the manifold along which all agents have the same opinion. Nevertheless, one can observe that the function $x_{\min }(t)=\min _{i=1, \ldots, N} x_{i}(t)$ is a nondecreasing function for the uncontrolled dynamics, and similarly $x_{\max }(t)=\max _{i=1, \ldots, N} x_{i}(t)$ is nonincreasing. As a consequence, the hypercube

$$
A=\left\{\left(x_{1}, \ldots, x_{N}\right) \in \mathbb{R}^{N} \mid x_{i} \in\left[x_{\min }(0), x_{\max }(0)\right]\right\}
$$

\footnotetext{
${ }^{1}$ For some configurations, the system has no unique solution, since the right-hand side is discontinuous with respect to the state variable.
} 
is an invariant subset for the uncontrolled dynamics. Moreover, we will define controls for which such a subset keeps being invariant under (18). One can then define a proper functional, coinciding with $V$ on such hypercube. For simplicity of notation, we denote it again by $V$. The subset $\Omega$ then coincides with the intersection of the consensus manifold $C$ with $A$.

We now present some numerical simulations for such a system. We consider 50 agents coming from a sampling of the uniform random variable on the interval $[0,10]$ as the initial data, and we apply the three strategies presented above, with different choices of the parameters. The results are presented in Figures 6, 7, 8, respectively for the three strategies, and for several values of $\tau$ and $\varepsilon$. Blue trajectories show the dynamics of uncontrolled agents, while red trajectories show trajectories on which the control is active. Red circles show configurations in which the controlled agent switches. Note that Strategies 2 and 3 outperform Strategy 1, providing faster convergence to consensus, since they act on extremal agents only.

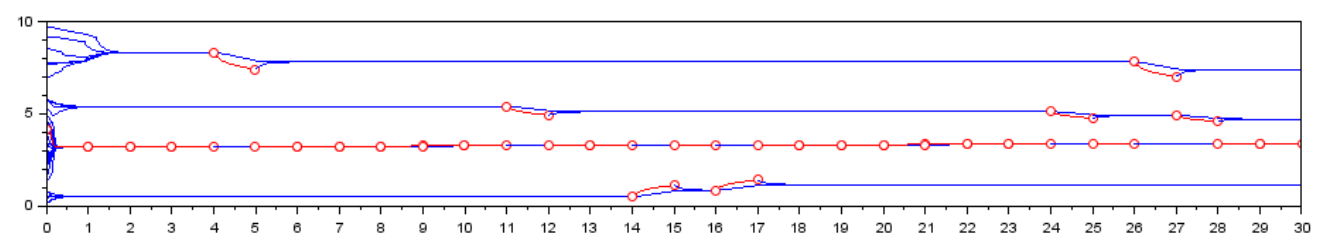

(a) $\tau=1$

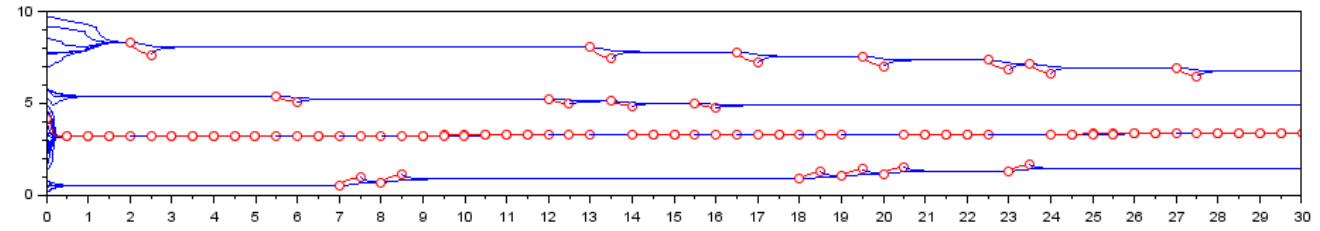

(b) $\tau=0.5$

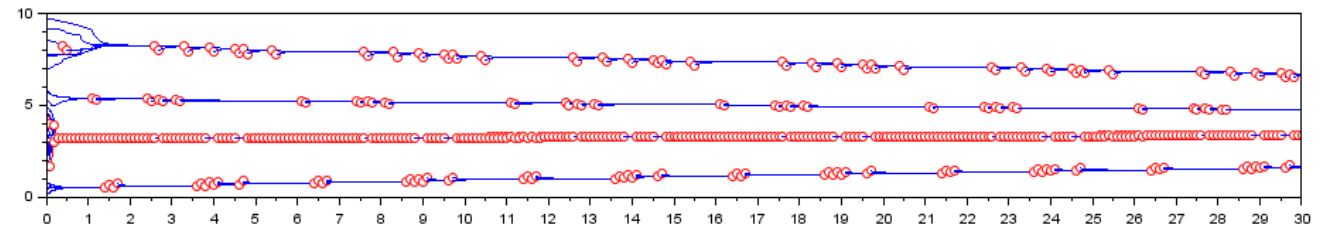

(c) $\tau=0.1$

Figure 6: Application of Strategy 1 to (18).

\section{References}

[1] Giacomo Albi, Mattia Bongini, Emiliano Cristiani, and Dante Kalise. Invisible control of self-organizing agents leaving unknown environments. SIAM Journal on Applied Mathematics, 76(4):1683-1710, 2016.

[2] Giacomo Albi and Lorenzo Pareschi. Modeling of self-organized systems interacting with a few individuals: from microscopic to macroscopic dynamics. Applied Mathematics Letters, 26(4):397401, 2013. 


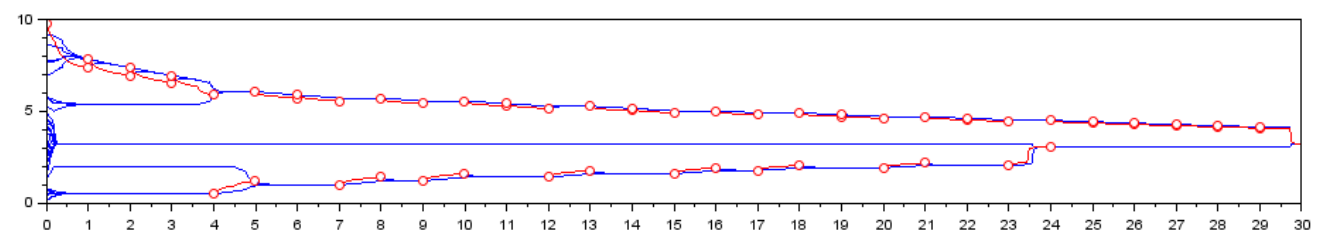

(a) $\tau=1$

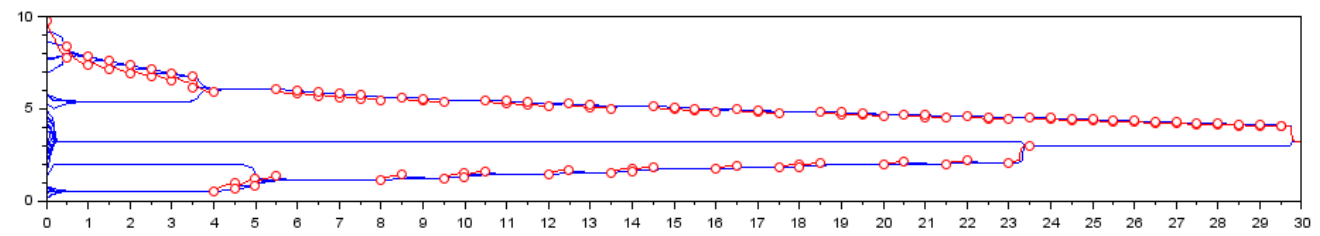

(b) $\tau=0.5$

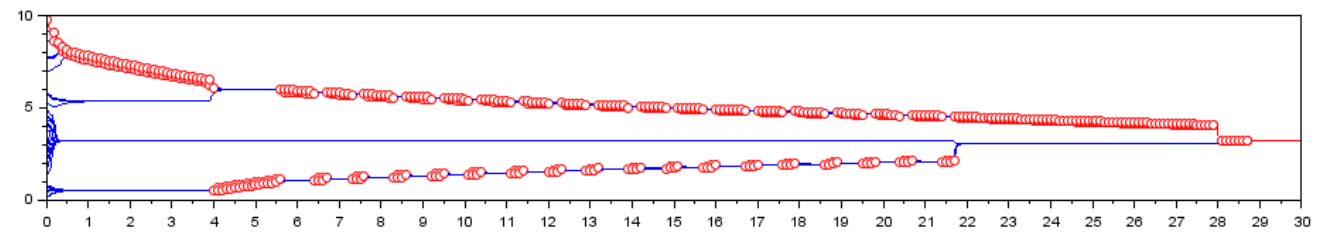

(c) $\tau=0.1$

Figure 7: Application of Strategy 2 to (18).

[3] Pierre Auger, Rachid Mchich, Tanmay Chowdhury, Gauthier Sallet, Maurice Tchuente, and Joydev Chattopadhyay. Effects of a disease affecting a predator on the dynamics of a predator-prey system. Journal of theoretical biology, 258(3):344-351, 2009.

[4] Vincent D Blondel, Julien M Hendrickx, and John N Tsitsiklis. Continuous-time averagepreserving opinion dynamics with opinion-dependent communications. SIAM Journal on Control and Optimization, 48(8):5214-5240, 2010.

[5] Mattia Bongini, Massimo Fornasier, Fabian Fröhlich, Laleh Haghverdi, et al. Sparse stabilization of dynamical systems driven by attraction and avoidance forces. Networks and Heterogeneous Media, 9(1):1-31, 2014.

[6] Mattia Bongini, Massimo Fornasier, Francesco Rossi, and Francesco Solombrino. Mean-Field Pontryagin Maximum Principle. submitted.

[7] Alfio Borzi and Suttida Wongkaew. Modeling and control through leadership of a refined flocking system. Mathematical Models and Methods in Applied Sciences, 25(02):255-282, 2015.

[8] Roger Brockett. Asymptotic stability and feedback stabilization. Differential geometric control theory, 27(1):181-191, 1983.

[9] Marco Caponigro, Massimo Fornasier, Benedetto Piccoli, and Emmanuel Trélat. Sparse stabilization and optimal control of the Cucker-Smale model. Mathematical Control and Related Fields, 3(4):447-466, 2013. 


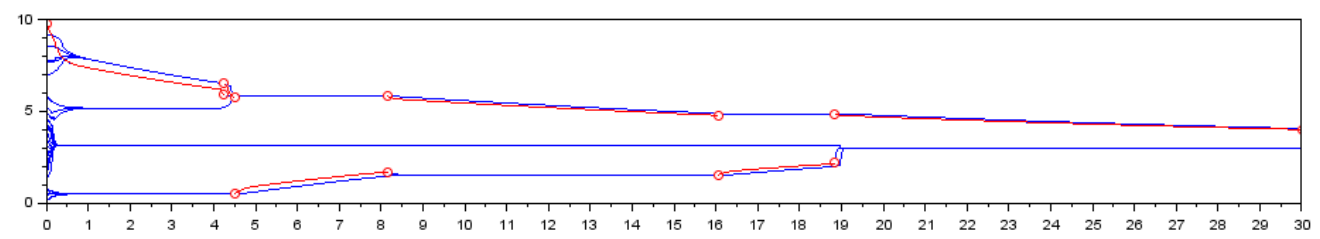

(a) $\varepsilon=0.2$

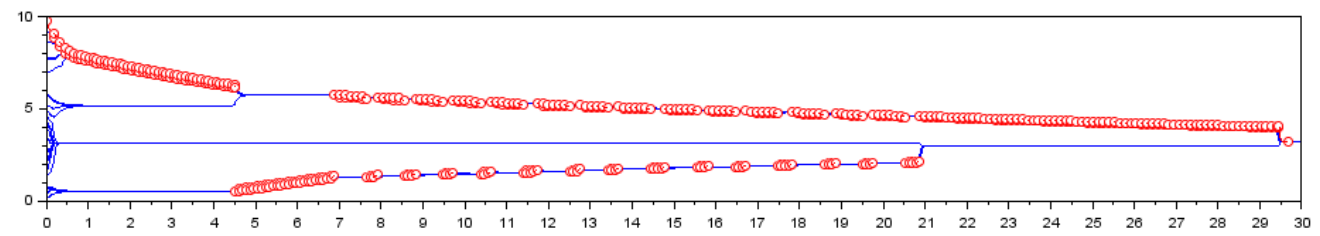

(b) $\varepsilon=0.05$

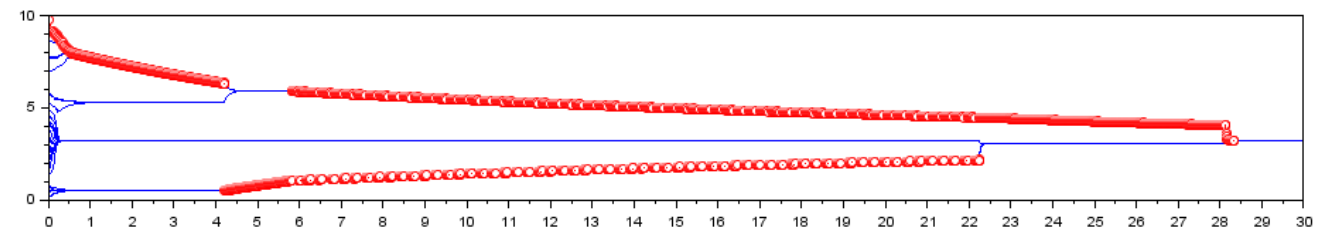

(c) $\varepsilon=0.01$

Figure 8: Application of Strategy 3 to (18).

[10] Marco Caponigro, Massimo Fornasier, Benedetto Piccoli, and Emmanuel Trélat. Sparse stabilization and control of alignment models. Mathematical Models and Methods in Applied Sciences, 25(03):521-564, 2015.

[11] Marco Caponigro, Benedetto Piccoli, Francesco Rossi, and Emmanuel Trélat. Sparse feedback stabilization of multi-agent dynamics. In Decision and Control (CDC), 2016 IEEE 55th Annual Conference on, 2016.

[12] Francis Clarke. Lyapunov functions and discontinuous stabilizing feedback. Annual reviews in control, 35(1):13-33, 2011.

[13] Francis H. Clarke, Yuri S. Ledyaev, Eduardo D. Sontag, and Andrei I. Subbotin. Asymptotic controllability implies feedback stabilization. IEEE Trans. Automat. Control, 42(10):1394-1407, 1997.

[14] Jean-Michel Coron. Global asymptotic stabilization for controllable systems without drift. Mathematics of Control, Signals and Systems, 5(3):295-312, 1992.

[15] Jean-Michel Coron. Control and nonlinearity. Number 136. American Mathematical Soc., 2007.

[16] Felipe Cucker and Jiu-Gang Dong. A conditional, collision-avoiding, model for swarming. Discrete and Continuous Dynamical Systems, 34(3):1009-1020, 2014.

[17] Florian Dietrich, Samuel Martin, and Marc Jungers. Opinion dynamics control by leadership with bounded influence. In Decision and Control (CDC), 2016 IEEE 55th Annual Conference on, 2016. 
[18] Ludovic Faubourg and Jean-Baptiste Pomet. Design of control Lyapunov functions for "JurdjevicQuinn" systems. In Stability and Stabilization of Nonlinear Systems, pages 137-150. Springer, 1999.

[19] Ludovic Faubourg and Jean-Baptiste Pomet. Control Lyapunov functions for homogeneous "Jurdjevic-Quinn" systems. ESAIM: Control, Optimisation and Calculus of Variations, 5:293$311,2000$.

[20] Massimo Fornasier, Benedetto Piccoli, and Francesco Rossi. Mean-field sparse optimal control. Philosophical Transactions of the Royal Society of London A: Mathematical, Physical and Engineering Sciences, 372(2028):20130400, 2014.

[21] Rainer Hegselmann and Ulrich Krause. Opinion dynamics and bounded confidence models, analysis, and simulation. Journal of Artificial Societies and Social Simulation, 5(3), 2002.

[22] Velimir Jurdjevic and John P Quinn. Controllability and stability. Journal of differential equations, 28(3):381-389, 1978.

[23] Michael Malisoff and Frédéric Mazenc. Constructions of strict Lyapunov functions. Springer Science \& Business Media, 2009.

[24] L. Moreau and D. Aeyels. Practical stability and stabilization. IEEE Transactions on Automatic Control, 45(8):1554-1558, Aug 2000.

[25] Sebastien Motsch and Eitan Tadmor. A new model for self-organized dynamics and its flocking behavior. Journal of Statistical Physics, 144(5):923-947, 2011.

[26] Grégoire Nicolis and Ilya Prigogine. Self-organization in nonequilibrium systems: From dissipative structures to order through fluctuations. NY: Wiley, 1977.

[27] R. Outbib and G. Sallet. Stabilizability of the angular velocity of a rigid body revisited. Systems and Control Letters, 18(2):93 - 98, 1992.

[28] Lionel Rosier and Jean-Michel Coron. A relation between continuous time-varying and discontinuous feedback stabilization. J. Math. Syst. Estim. Control, 4:67-84, 1994.

[29] Claude Samson. Velocity and torque feedback control of a nonholonomic cart, pages 125-151. Springer Berlin Heidelberg, Berlin, Heidelberg, 1991.

[30] J. A. Sanders, F. Verhulst, and J. Murdock. Averaging methods in nonlinear dynamical systems, volume 59 of Applied Mathematical Sciences. Springer, New York, second edition, 2007.

[31] E. Sontag and H. Sussmann. Remarks on continuous feedback. In Decision and Control including the Symposium on Adaptive Processes, 1980 19th IEEE Conference on, pages 916-921, Dec 1980.

[32] Andrew R. Teel, Joan Peuteman, and Dirk Aeyels. Semi-global practical asymptotic stability and averaging. Systems \& Control Letters, 37(5):329 - 334, 1999.

[33] Suttida Wongkaew, Marco Caponigro, and Alfio Borzi. On the control through leadership of the Hegselmann-Krause opinion formation model. Mathematical Models and Methods in Applied Sciences, 25(03):565-585, 2015. 
[34] Jiamin Zhu, Emmanuel Trélat, and Max Cerf. Planar tilting maneuver of a spacecraft: singular arcs in the minimum time problem and chattering. Discrete Contin. Dyn. Syst. Ser. B, 21(4):1347$1388,2016$. 